\title{
Rencontre avec Marcel Maréchal, du Théâtre National de Marseille
}

\section{Marcel Maréchal}

\section{(2) OpenEdition \\ Journals}

\section{Édition électronique}

URL : http://journals.openedition.org/shakespeare/505

DOI : 10.4000/shakespeare.505

ISSN : 2271-6424

\section{Éditeur}

Société Française Shakespeare

\section{Édition imprimée}

Date de publication : 1 novembre 1984

Pagination : 169-170

\section{Référence électronique}

Marcel Maréchal, «Rencontre avec Marcel Maréchal, du Théâtre National de Marseille », Actes des congrès de la Société française Shakespeare [En ligne], 5 | 1984, mis en ligne le 01 janvier 2007, consulté le 06 mai 2019. URL : http://journals.openedition.org/shakespeare/505 ; DOI : 10.4000/ shakespeare.505 
SOCIETE FRANÇAISE SHAKESPEARE

Actes du Congrès 1983

\section{MYTHE ET HISTOIRE}

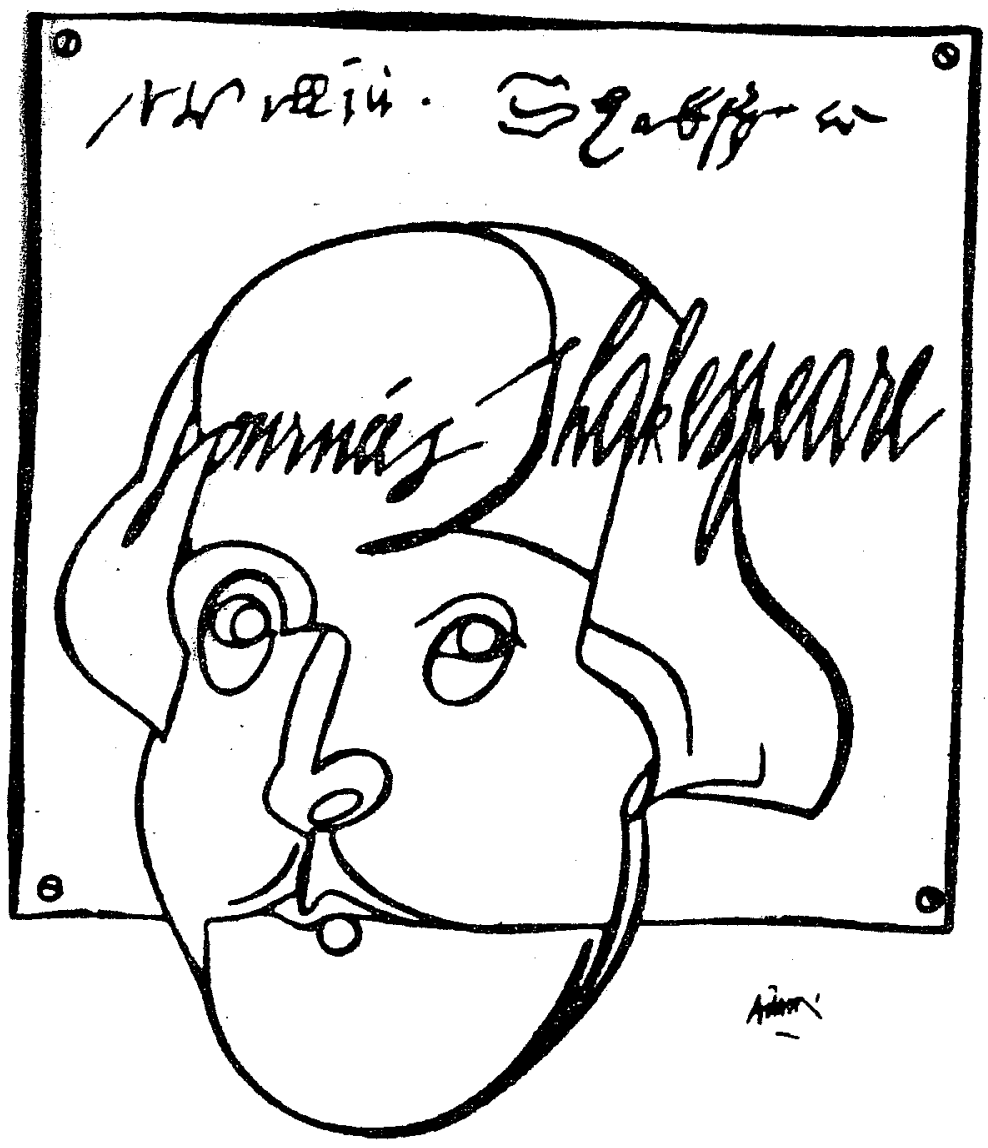

$$
\begin{array}{r}
\text { DiReCteur DE LA' PUblicatic } \\
\text { M.T. Jones-Davie }
\end{array}
$$

Publié avec le concours du Centre National de la Recherche Scientifiq

$$
\text { JEAN TOUZOT Libraire - Editeur }
$$

38, rue Saint-Sulpice 75278 PARIS CEDEX 06199 


\section{Entretien avec Marcel MARECHAL \\ (Théâtre de la Criée de Marseille) \\ sur Le Roi Lear (création : janvier 1984) \\ animé par J. M. DEPRATS}

Marcel Maréchal a déjà monté plusieurs pièces de Shakespeare et du répertoire élisabéthain : Tamerlan, Roméo et Juliette (1970-1971), Hamlet, Falstaff (d'après 1 et 2 Henry IV), (1975-1976), Skakespeare, notre contemporain (montage 1966-1967), Le Sang, de Jean Vauthier, d'après La Tragédie du Vengeur de C. Tourneur.

Marcel Maréchal se déclare plus à l'aise dans l'univers shakespearien que dans celui de Molière, car Shakespeare lui paraît offrir plus de possibilités d'ouverture sur le monde d'aujourd'hui. S'agissant du Roi Lear, il ne souhaite pas formuler de déclaration d'intentions, car son travail sur la pièce ne fait que commencer.

\section{- Quel texte français allez-vous utiliser ?}

$M$. $M$. La traduction de Jean Vauthier, également adaptateur de Roméo et Juliette et d'Othello. Cette traduction est encore inédite.

- Cette traduction comporte-t-elle une différence de style pour la prose et les vers?

$M . M$. Il s'agit d'une version française pour la scène et non d'une adaptation libre. Vauthier a respecté la musique du vers, mais ne s'est pas attaché à marquer des différences. Le texte est très sonore et agréable pour l'acteur. Le traducteur a évité les périphrases et s'est efforcé de trouver des correspondances de mots, en recourant parfois aux archaïsmes.

- Vous paraissez avoir opté pour la médiation d'un poète. Récusez-vous les traductions littérales?

$M$. $M$. 'Oui, la médiation d'un poète, d'un créateur, est nécessaire (Vauthier, Novarina). Pour que le texte «parvienne» au public, il faut que le traducteur fasse oeuvre poétique, ce qui n'est pas toujours le cas dans les éditions bilingues. Le metteur en scène doit réfléchir sur le texte 
avec le médiateur-poète : il faut que le public saisisse bien, car le moment du spectacle est éphémère.

Marcel Maréchal fait la lecture d'un passage de King Lear, acte III, scène ii.

- C'est plus qu'une traduction : c'est une réécriture. La collaboration d'un angliciste serait nécessaire pour garantir le sens. En outre, la question du texte utilisé se pose désormais, car on considère à présent qu'il y a deux King Lear. Une collaboration tripartite (acteurs, poète, spécialiste d'élisabéthain) serait souhaitable.

$M . M$. C'est une réécriture. Le texte est assez porteur pour le comédien, même s'il y a quelques fautes. Vauthier a travaillé à travers le mot à mot d'un angliciste.

- Quel choix de costumes envisagez-vous : passé reculé, présent intemporel, présent déterminé, mais symbolique ? (référence à la représentation de Tamerlan à Arras en costumes Mao).

$M$. M. Tamerlan a été joué dans la traduction de Michel Parent. Les costumes ont servi à montrer un personnage rigoureux malgré sa grande sensualité, effectuant une «longue marche». Les costumes seront sans actualisation : cuirs, fourrures, drap.

- Et le décor?

$M . M$. Ce sera le «théâtre du monde»: un théâtre apparent, échoué de travers sur la scène (théâtre dans le théatre, emboîtement), plus ou moins entouré d'eau. Il s'ouvrira peu à peu sur le monde pour aboutir à l'épure de la scène finale (rédemption). Le Roi Lear se situe dans un univers du dedans et un univers du dehors qui s'opposent (portes qui s'ouvrent et qui se ferment).

Lecture d'un passage d'Yves Bonnefoy sur la stratégie de King Lear comme «acte de foi où le sens survit», ou des «fils d'acier sont tendus sur un affreux abîme»

M. M. Hamlet est plus pessimiste que King Lear. Les scènes de la fin renferment une valeur rédemptrice : la pièce est porteuse d'espoir. On ne peut évacuer le pessimisme mis en évidence par Jan Kott, mais l'interprétation 
peut aller vers une aube.

- Le roi Lear perd tout pour devenir pur; les anciennes valeurs ont disparu.

M. M. Oui. La fin doit être jouée comme un chant.

- Les dernières paroles de Lear sont ambiguës : meurt-il de joie ou de désespoir? Un acteur peut-il faire passer cette incertitude dans son interprétation?

M. M. Oui, je pense que c'est possible.

- Mettez-vous en évidence les deux dénouements (le tournoi : bon dénouement ; la mort de Cordelia et de Lear : dénouement tragique) ? Le vrai dénouement n'est pas dans la restauration de l'ordre, mais dans la destructuration. Comment utilisez-vous le duel? tiel.

$M . M$. De façon très fulgurante, pour aller à l'essen-

- Comment traiterez-vous la violence?

$M . M$. Je n'ai pas de réponse toute faite. Tout dépend du rapport que l'époque de la représentation entretient avec la violence.

- Tout dépend de la présentation ; à Stratford, les effets de mise en scène rendaient le "Lear» de Bond plus tragique que celui de Shakespeare. Comment présenterez-vous la scène du «suicide» de Gloucester?

$M . M$ Il n'est pas genant que certains passages fassent rire. La chute de Gloucester est celle du pitre qui tombe sur un plancher. On peut aussi aveugler les spectateurs pendant que Gloucester tombe.

- Il faudrait ménager un temps pendant lequel Gloucester resterait inanimé. Le rire de la chute anéantit l'émotion.

M. M. Il faut qu'Edgar soit au centre. Le rôle doit être joué par un comédien de grande race : il est plus difficile d'être éloquent dans une solution de l'ordre de la feinte.

Partagez-vous bien Lear et Gloucester ou, comme' chez Strehler, y-a-t-il confusion entre les deux personncages?

M. M. Non, et pas de confusion non plus entre Cordelia et le Fou. Il est réducteur de confondre Lear et Glou- 
cester : Lear est plus loin.

- Qu'est-ce que le Fou pour un public d'aujourd'hui?

M. M. C'est un rôle très dur. Daniel Berliot, qui joue ce rôle, va répéter Lear à ma place et ensuite, je répèterai seul avec lui. Je pense qu'une connivence naîtra. C'est un jeune acteur qui a des moyens vocaux. Le Fou, dans Lear, c'est le vrai fils. J'aimerais ne pas le faire disparaître : rien ne l'interdit. Dans une mise en scène russe, il jouait de la flûte dans la dernière scène avec Cordelia.

- Quel costume portera-t-il ?

$M$. $M$. Il aura un grand manteau charlotesque et un chapeau insolite, lui donnant l'allure d'un clochard. Mais son costume pourra être remis en cause jusqu'au dernier moment.

- Quel Lear choisirez-vous ? Marvin Rosenberg nous a montré, il y a quelques années, quatre interprétations possibles (sénile, chef de guerre, fou, colérique) de la première scène.

$M . M$. Il ne sera pas sénile, mais violent et très fou. C'est à ce moment qu'il est le plus fou. Je prendrai les signes de vieillissement, mais pas la barbe. Ensuite Lear aura la tête rasée (dépouillement).

- Couperez-vous la scène du jugement?

$M . M$. Non, c'est une belle scène.

- Le procès parodique est le théâtre de Lear qui lui permet de dominer une situation à l'intérieur de cadres théâtraux. De même dans la scène de la falaise, Gloucester est le spectateur aveugle sur le micro-théâtre d'Edgar.

$M . M$. Mettre en scène une coupure éventuelle peut être profitable : le non-joué subsiste dans le joué.

Le Roi Lear sera joué en mars 1984 au Théâtre de Paris. 